\title{
DFT-based Cluster Analysis Channel Estimation Algorithm for OFDM Systems on Multipath Channels
}

\author{
Tongliang Fan, Yucang Wen, Xinli Ma, Yongfeng Du and Mo Li \\ Electronic Technology Department, China Maritime Police Academy, Ningbo, \\ 315801, China \\ libufan432@163.com
}

\begin{abstract}
In this paper, a enhanced DFT-based channel estimation for OFDM systems is proposed. Conventional DFT-based channel estimations improve the performance by suppressing time domain noise beyond the length of the cyclic prefix $(C P)$, so it does not completely suppress the noise. However, they potentially require information on channel impulse responses and may also result in mean-square error (MSE) floor due to incorrect channel information such as channel delay spread. In order to overcome the disadvantage, our proposed channel estimation can improve the performance by deciding significant channel taps adaptively. Significant channel taps are detected on the basis of cluster discriminant analysis. Simulation results demonstrate that the proposed algorithm outperforms the conventional DFT-based estimation in terms of BER and MSE performance.
\end{abstract}

Keywords: OFDM; DFT-based channel estimation; CIR (Channel Impulse Response); cluster analysis

\section{Introduction}

Orthogonal frequency division multiplexing (OFDM) has many well known advantages such as robustness in frequency selective fading channels, high bandwidth efficiency, and so on. OFDM has been widely applied to wireless communication system and broadcasting. For coherent OFDM systems, a robust channel estimator is required. To achieve this, several channel estimation methods are studied. In general, The LS estimator is the simplest channel estimation [1]. The minimum mean-square error MMSE [2] estimator shows good performance. But it requires knowledge of auto-covariance matrix of the multi-path fading channels, thus its computational complexity is very high. By partitioning off channel covariance matrix into some small matrices on the basis of coherent bandwidth, Noh et al., [3] proposed a low-complexity LMMSE estimation method. However, the modified LMMSE methods still have high-computational complexity. For this reason, Zhao et al., [4] introduced additional DFT processing to obtain the frequency response of LS estimated channel. Dowler et al., [5] showed the performance of various channel estimation methods and concluded that the DFT-based estimator can achieve significant performance benefits if the maximum channel delay is known. Minn et al., [6] improved on this idea by considering only the most significant channel taps. Similarly, Athaudage et al., [7] proposed a delay spread estimation method using cyclic prefix (CP) that can be useful to improve the DFT-based estimation. You et al., [8] proposed a channel estimation method based on a time-domain threshold which is the standard deviation of noise obtained by wavelet decomposition. The threshold value becomes a crucial factor, so it is required to find an optimal threshold value to obtain the desired results. 
By suppressing time domain noise, conventional DFT-based channel estimations improve the performance. However, they potentially require the length information of CIR. To compensate for the vulnerability to noise of the LS estimator and increase the estimation accuracy of conventional DFT-based channel estimator, Fan et al., [9] presented discriminant analysis for channel estimation in time domain. But, it requested a large amount of statistical data, which is very difficult to obtain in practice.

Since CIR is not longer than the guard interval (GI) in OFDM system, the CIR longer than the GI length can be regarded as noise. Removing the noise and identify channel taps from channel impulse response (CIR), it can be regarded as a problem of pattern recognition. The cluster analysis algorithm is a classical analytic method of pattern recognition. The cluster structure derived from clustering techniques is used to distinguish channel taps from the noise, and it is done on the basis of similarities. In this paper, we propose a modified DFTbased channel estimation algorithm based on cluster analysis to reduce residual noise existing at the estimated channel coefficients in time domain. Simulation results demonstrate that the proposed algorithm outperforms the conventional DFT-based estimation in terms of BER and MSE performance.

The rest of this paper is organized as follows. In Section 2 we briefly go over the OFDM system model. In Section 3, we provide a review of conventional DFT-based channel estimation algorithms. A modified DFT-based channel is proposed in Section 4. Simulation results of mean square error (MSE) and bit error rate (BER) are presented in Section 5. The final conclusions are given in Section 6.

\section{OFDM System Model}

We consider an OFDM system that consists of $N$ sub-carriers. And each sub-carrier consists of data symbol $X[k]$, where $k$ represents the sub-carrier index. The OFDM transmitter uses an inverse DFT of size $N$ for modulation. Then the transmitted OFDM signals in discrete-time domain can be expressed as:

$$
x[n]=\frac{1}{N} \sum_{k=0}^{N-1} X[k] \exp \left(j 2 \pi \frac{n k}{N}\right), \quad 0 \leq n \leq N-1 .
$$

Where $n$ is the time domain sample index of an OFDM signals. In order to avoid ISI caused by multipath channel and consequent ICI, GI is appended to the beginning of the $N$ sample of IDFT. After passing through a time-varying multi-path fading channel and removing the GI, one received signal $y[n]$ consists of channel distorted versions of the transmitted OFDM symbols, which is represented by

$$
\mathrm{y}[\mathrm{n}]=x[n] \otimes h[n]+w[n] \quad 0 \leq n \leq N-1 .
$$

Where $\otimes$ denotes cyclic convolution operation, $w[n]$ is i.i.d additive white Gaussian noise (AWGN) sample in time domain with zero mean and variance $\sigma_{w t}^{2}$ and $h[n]$ is the discrete-time CIR given by

$$
\sigma_{w t}^{2}=E\left[|w[n]|^{2}\right] \text { and } h[n]=\sum_{l=0}^{L-1} \alpha_{l} \delta\left[n-\tau_{l}\right]
$$


Where $\alpha_{l} \quad l=1,2, \cdots, L$ represents a different path complex gain at $\tau_{l}$ sample, $\tau_{l}$ is integer number normalized by channel delay, which means there is no channel power loss caused by sampling time mismatch [8] and $L$ is number of multi-path. Usually, $\alpha_{l}$ is modeled as complex Gaussian processes with Jakes' power spectrum, and all the delay paths are uncorrelated to each other. At the receiver, we assume that the guard interval is longer than the maximum channel delay and the synchronization is perfect. Then, the received signal in frequency domain can be represented by

$$
Y[k]=X[k] H[k]+W[k], \quad 0 \leq k \leq N-1 .
$$

Where $W[k]$ is AWGN samples in frequency domain with zero mean and variances $\sigma_{w f}^{2}=N \sigma_{w t}^{2}[10]$ and $H[k]$ is channel frequency response that is DFT of CIR $h[n]$ with length $L$ and can be expressed as:

$$
H[k]=\sum_{l=0}^{L-1} \alpha_{l} \exp \left(-j 2 \pi \frac{n l}{N}\right) \quad 0 \leq k \leq N-1 .
$$

It is shown in (4) that, to detect the transmitted data $X[k]$, the receiver needs to estimate the channel gain $H[k]$.

\section{Channel Estimation Algorithm}

\subsection{LS Channel Estimation}

At the receiver, the received pilot signals are extracted from $Y[k]$. For the pilot subcarriers, the transmitted information $X[k]$ is known. Then the channel frequency response in pilot sub-channels can be estimated by:

$$
\hat{H}_{L S}[k]=\frac{Y[k]}{X[k]}=H[k]+\frac{W[k]}{X[k]} \quad k \in K_{P} .
$$

This channel estimation algorithm is called the LS estimator, where $K_{P}$ is the ensemble of all pilot indexes. The greatest advantage of LS estimation algorithm is its simple structure and lowly complexity. Since LS does not make use of any channel information. As a result, the accuracy of this algorithm is limited; LS algorithm is useful when channel noise is small.

The LS estimation has a large MSE, which does not guarantee a satisfactory performance. The individual MSE of $k$ th sub-carrier [11] is

$$
M S E_{L S}[k]=\frac{\beta}{S N R} .
$$

Where $S N R=E\left[|w[k]|^{2}\right] / \sigma_{w f}^{2}$ is the average SNR and $\beta=E\left[|w[k]|^{2}\right] E\left[|w[k]|^{-2}\right]$ is a constant depending on signal constellation.

\subsection{DFT-based Channel Estimation}

DFT-based channel estimator is a method for CIR estimation by transforming the frequency domain estimates into time domain estimates. Since a large number of time-domain channel power concentrate on a few first samples, the DFT-based estimation reduces the 
noise power that exists only outside of the CIR part. The $n$th estimated sample of CIR is expressed as:

$$
\hat{h}_{L S}[n]=I D F T\left\{\hat{H}_{L S}[k]\right\}=h[n]+\tilde{w}[n] \quad 0 \leq n \leq N-1 .
$$

Where $I D F T\{\bullet\}$ indicates N-point IDFT. $\tilde{w}[n]$ is IDFT sample of noise, which is also AWGN because IDFT is a linear transform. The CIR is typically limited to the length of CIR $L$ which is less than the GI and much smaller compared with the number of sub-carrier $N$. Conventionally, the CIR is described as:

$$
h[n]=\left\{\begin{array}{lc}
I D F T_{N}\{H[k]\}, & 0 \leq n \leq L-1 \\
0, & L \leq n \leq N-1
\end{array} .\right.
$$

By using (9) and (8) $\hat{h}_{L S}[n]$ can be divided into two parts: CIR part and noise-only existing part, then we have

$$
\hat{h}_{L S}[n]=\left\{\begin{array}{lc}
h[n]+\tilde{w}[n], & 0 \leq n \leq L-1 \\
\tilde{w}[n], & L \leq n \leq N-1
\end{array} .\right.
$$

As shown in (9), all CIR existed in the first $L$ samples and other samples are only noise. Because the $L$ is unknown, by zero-padding for pure noise period, the conventional DFTbased estimator can be expressed as:

$$
\hat{h}_{D F T}[n]=\left\{\begin{array}{lc}
h[n]+\tilde{w}[n], & 0 \leq n \leq L-1 \\
0, & L \leq n \leq N-1
\end{array} .\right.
$$

From (11), the DFT-based channel estimation is denoted as

$$
\hat{H}_{D F T}[k]=D F T_{N}\left\{\hat{h}_{D F T}[n]\right\} \quad 0 \leq k \leq N-1 .
$$

In absence of additive noise, it is easy to show that the DFT-based estimator can perfectly recover the channel frequency response of all sub-carriers. With assumption that channels have sample-spaced impulse, the individual MSE of DFT-based channel estimation [9] is given as

$$
M S E_{D F T}[k]=\frac{N_{c p}}{N} \frac{\beta}{S N R}
$$

Where $\mathrm{N}$ is the number of sub-carriers and $N_{c p}$ is the length of GI. In this case, the MSE of conventional DFT-based channel estimation is fixed, even though the real samples are much smaller than length of GI. On the contrary, if we adaptively choose $L$ as the length of the present channel, we can expect the better performance. Hence, we propose an improved estimation algorithm in the next section on the basis of this idea.

\section{Proposed Channel Estimation}

Usually, the performance of conventional DFT-based estimation depends on the estimation accuracy of path delay. Instead of estimating channel path delay, we focus on decision the 
significant channel taps with CIR adaptor. There have been some useful threshold values for the use of noise reduction. Most of them are highly associated with the energy of the noise added to the signal. One of available threshold values can be determined by estimating the maximum channel tap's energy. As shown in equation (10), the estimated channel taps are composed of the ideal channel taps and the added noise spectrum. Usually, $L$ is an unknown variable depending on the channel environments? To overcome the weakness of DFT-based estimator and estimate an unknown CIR that varies according to channel environments, we focus on determining the significant channel taps with CIR part. In this section, we propose an improved estimation algorithms based on cluster analysis.

\subsection{Cluster Analysis}

Cluster analysis is an unsupervised learning method, and a common technique for data analysis used in many fields. Cluster analysis is the task of assigning a set of objects into groups (called clusters) so that the objects in the same cluster are more similar to each other than to those in other clusters. Clustering is based on the core idea of objects being more related to nearby objects than to objects farther away. A cluster can be described largely by the maximum distance needed to connect core of the cluster.

In the channel estimator, the LS estimator is subject to noise. To overcome the vulnerability of the LS method, our research is concerned with noise suppression in timedomain. We suppose that the duration of the CIR is shorter than the GI. It means that the length $L$ of CIR is shorter than the length $N_{C P}$ of CP and much shorter compared with the number of sub-carrier $N$. Whereas the noise term $\tilde{w}[n]$ in equation (8) is represented in the whole time domain. Consequently, while $N_{C P} \leq n \leq N-1$, the element of $\hat{h}_{L S}[n]$ in equation (8) are all to be handled estimation noise, and all of these elements are regarded as noise cluster. From these date sets, the core $\bar{w}$ of noise cluster can be calculated by the following equation:

$$
\bar{w}=\frac{1}{N-L} \sum_{i=L}^{N-1} \hat{h}_{L S}(i)
$$

The cluster function can be got from calculating the max distance $r_{w}$ between $\hat{h}_{L S}[n]$ and $\bar{w}$ in the region $N_{C P} \leq n \leq N-1$. It can be used to identify the channel taps and noise in the interval $0 \leq n \leq N_{C P}-1$.

The process consists of the following steps:

Step1: Compute $\hat{H}_{L S}[k]$ from the equation (7);

Step2: Compute $\hat{h}_{L S}[n]$ from the equation (8).

Step3: In the region $N_{C P} \leq n \leq N-1$, calculate the core $\bar{w}$, find the absolute maximum value $\hat{h}_{\text {noise } \text { max real }_{-}}$of real and $\hat{h}_{\text {noise_ } \text { maximag }_{\text {mag }}}$ of imaginary part of CIR, separately. Let $\hat{h}_{L S}[n]=0$. 
Step4: Let $h_{\text {noise } \text { max }_{\text {max }}}=\hat{h}_{\text {noise_max real }}+i * \hat{h}_{\text {noise_maximag }}$. Computer the cluster threshold function $r_{w}=\left\|h_{\text {noise } \text { max }_{\text {max }}}-\bar{w}\right\|$ identify the noise in the region $0 \leq n \leq N_{C P}-1$. If $\left\|\hat{h}_{L S}[n]-\bar{w}\right\| \leq r_{w}, \hat{h}_{L S}[n]=0$.

Step5: Take FFT of the resulting of step4.

We name our method as Cluster analysis channel estimation based on DFT, and it can be abbreviated as DFT-CAT.

The individual MSE of $k$ th sub-carrier is:

$$
M S E_{D F T}[k]=\frac{L}{N} \frac{\beta}{S N R}
$$

\section{Simulation Results}

In this section, we performed computer simulation to investigate the performance of the two proposed channel estimation algorithms. The MSE and BER performances are examined. The simulations are concentrated on comparison between LS algorithm, conventional DFT algorithm and both of the proposed algorithms. Let us briefly refer to the main parameters in the OFDM system that we consider. An OFDM system with symbols modulated by 16-QAM is used on multipath channels. The system bandwidth is $20 \mathrm{MHz}$, and the carrier frequency is $2.4 \mathrm{GHz}$, maximum Doppler frequency shift is $132 \mathrm{~Hz}$, the number of sub-carriers is 128 , and guard interval is $7.8125 \mathrm{kHz}$. Unit delay of channel is assumed to be the same as OFDM sample period. Thus, there are no power losses caused by non-sample spaced. Moreover, we assume that channels are static over one OFDM frame, and there are no synchronization error and no frequency offset at the receiver.

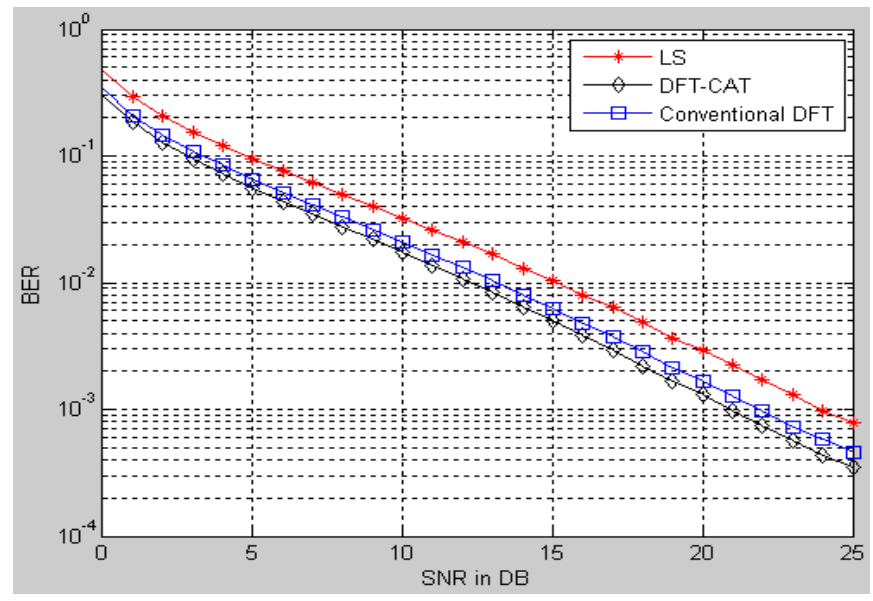

Figure 1. Comparison of the BER Performance

BER is used to measure the signal detection, which is the number of bit errors divided by the total number of transferred bits during a studied time interval. It can be counted in the simulation. Figure 1 shows the BER performances of the conventional DFT, the LS method and the proposed methods.

Through the comparison of the BER performance of three algorithms from Figure 2, with the increase of SNR, the bit error rate of the three kind algorithms are all gradually reduced. 
As shown in Figure 1, the DFT_CAT algorithm outperforms the existing methods. In the same SNR, the proposed methods are superior to the Conventional DFT.

Figure 2 shows the MSE of the channel estimation as a function of SNR for the three algorithms. Observe that the computer simulation is well verified by the theoretical result. The curve of the proposed algorithm has a fluctuation in the low signal to noise ratio. Because in the low SNR case, the noise of the CIR is similar with the channel of CIR and is difficult discriminate, thereby lead to error discriminate analysis.

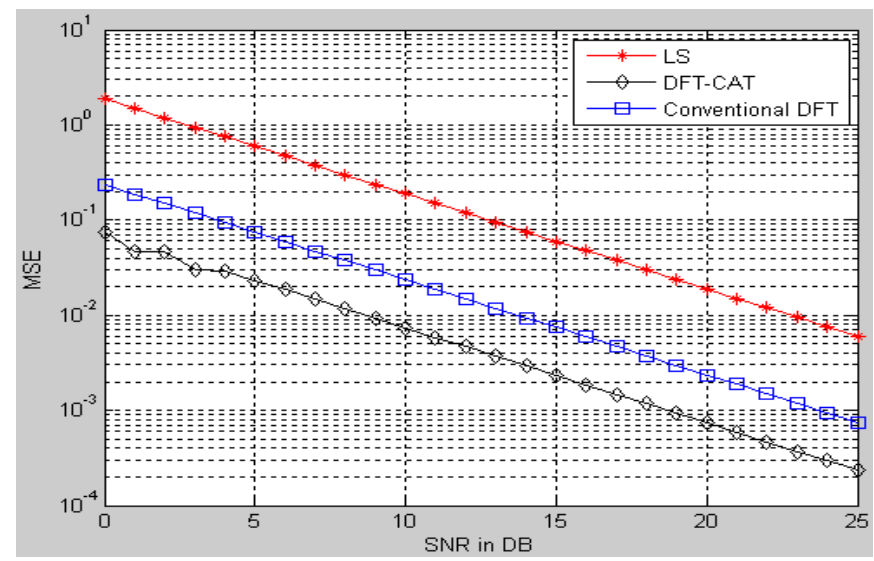

Figure 2. Comparison of the MSE Performance

\section{Conclusion}

In this paper, we proposed a noise-robust channel estimation method for OFDM systems based on cluster analysis. The estimated channel by the proposed method works as precisely as the known channel in terms of the BER. Adopting the proposed method in OFDM systems, we may effectively reduce estimation noise. It does not make MSE floor in any channels contrary to conventional DFT-based method. The simulation results show that the proposed algorithm can significantly reduce the MSE. In the future, we will increase the accuracy in the low SNR.

\section{Acknowledgements}

This work is supported by the research fund from China Maritime Police Academy (Grant No.2013XYPYZ012). The authors would like to acknowledge the anonymous reviewers for their thorough review and constructive suggestions, which significantly helped to improve the quality of the manuscript.

\section{References}

[1] M. Sandell Edfors and J.-J. Van, “OFDM Channel Estimation By Singular Value Decomposition”, IEEE Trans. Communication, vol. 46, no. 7, (1998) July, pp. 931-39.

[2] J.-J. van de Beek, O. Edfors, M. Sandell, S. K. Wilson and P. O. Börjesson, "On Channel Estimation in OFDM Systems”, IEEE 45th Vehicular Technology Conference, Chicago, vol. 2, (1995) July, pp. 815-819.

[3] Y. Zhao and A. Huang, "A Novel Channel Estimation Method for OFDM Mobile Communication Systems Based on Pilot Signals and Transform-Domain Processing”, Vehicular Technology Conference, Phoenix, AZ, (1997) May, pp. 2089-93.

[4] A. Dowler, A. Doufexi, and A. Nix, "Performance Evaluation of Channel Estimation Techniques for a Mobile Fourth Generation Wide Area OFDM System", IEEE 56th Vehicular Technology Conference, Canada, vol. 4, (2002) September, pp. 2036-40. 
[5] H. Minn and V. K. Bhargava, "An Investigation into Time-Domain Approach for OFDM Channel Estimation”, IEEE Transactions on, Broadcast, vol. 46, no. 4, (2000) December, pp. 240-48.

[6] C. R. N. Athaudage and A. D. S. Jayalath, "Delay-spread estimation using cyclic-prefix in wireless OFDM systems", IEE Proceedings-Communications, vol. 151, no. 6, (2004) pp. 559-566.

[7] Y. Lee, H. Shin, and H. Kim, "Channel Estimation Based on a Time-Domain Threshold for OFDM Systems", IEEE Transactions on, Broadcast, vol. 55, no. 3, (2009) September, pp. 656-663.

[8] M. James, J. Douglas and E. Paul, "Analyzing multivariate data”, Duxbury Pr., (2003).

[9] T. Fan, S. Yang, H. Wu and D. Wang, "Two Novel Channel Estimation for OFDM Systems by TimeDomain Cluster Discriminant Analysis Based on Parametric Channel Modeling”, Wireless PersCommun, vol 68, no. 2, (2013) January, pp 349-360.

[10] Y. Kang, K. Kim and H. Park, "Efficient DFT-based channel estimation for OFDM systems on multipath channels", IET. Communications, vol. 1, no. 2, (2007) April, pp. 197-202.

[11] H. Minn, and V. Bhargava, "An investigation into time-domain approach for OFDM channel estimation", IEEE Transactions on, Broadcast, vol. 64, no. 4, (2000), pp. 240-248.

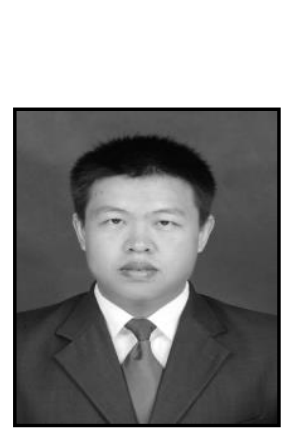

\section{Authors}

Tongliang Fan is currently a Lecturer at Electronic Technology Department, China Maritime Police Academy since June 2012. He obtained his $\mathrm{Ph}$. D. degree in the Center of Communication and Tracking Telemetry Command, Chongqing University, China, in June 2012. He obtained his M.S. in Chongqing University of Posts and Telecommunications and studied network management and network flow measurement from 2003 to 2006. He worked as an algorithm engineer with Chongqing CYIT Communication Technologies CL., LTD from 2006 until 2008. His research interest and activities include PHY layer design for terrestrial (4G) communications based on OFDM, antenna diversity techniques for wireless communications broadcast systems and positioning in wireless communications, shortwave and Ultrashort-wave in military communications.

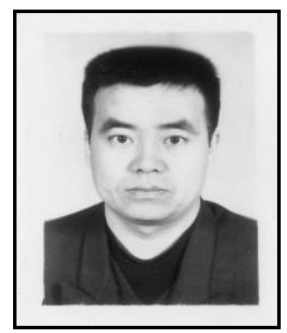

Yucang Wen is currently an associate professor at Electronic Technology Department, China Maritime Police Academy. He received the M.S. degree in technology and application of computer from Beijing institute of technology, china, 2003. He has been engaged in military communication research. His research interest and activities include technology and application of multimedia, computer technology and application, IP network communication and application.

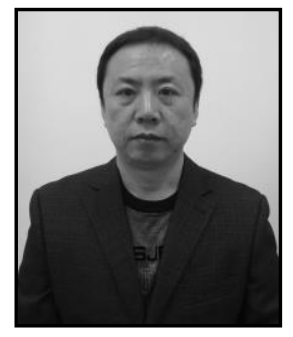

Xinli Ma is currently a professor at Electronic Technology Department, China Maritime Police Academy. He received the M.S. degree in Beijing science and engineering university, in 2004. His research interests and activities include short-wave communication, ultrashort wave communication, adaptive antenna and other communication technologies which are applied in military communication applications. He has published two monographs, four teaching materials, and thirty papers in various academic journals as well as in kinds of academic conferences. 


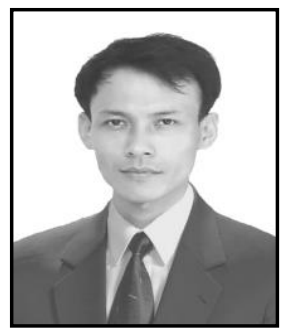

Yongfeng Du is currently a Lecturer at Electronic Technology Department, China Maritime Police Academy. He received the B.S. degree from Technical College of Armed Police Force (University of Chinese people's Armed Police Forces), in 1995. He had engaged in Communications technical support, Equipment maintenance and Communication service. His research interest and activities include video technology, signal processing and communication command.

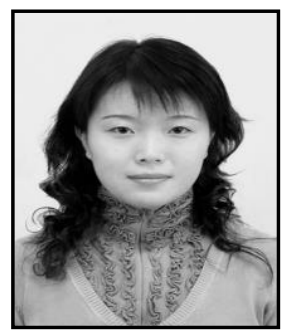

Mo Li was born in 1983. She received her B.E. and M.E. degree in Communication Engineering in 2005 and 2007, respectively, from Jilin University, China. She obtained her Ph. D. degree in PLA University of Science and Technology, China, in June 2011. She currently works in China Maritime Police Academy. Her research interests include Cognitive Radio, radio resource management and learning mechanism in the field of wireless communication. 
International Journal of Future Generation Communication and Networking Vol.7, No.1 (2014) 